\title{
Coulomb gauge studies of $S U(3)$ Yang-Mills theory on the lattice
}

\section{Aiko Voigt ${ }^{* a, b}$, Ernst-Michael Ilgenfritz ${ }^{a}$, Michael Müller-Preussker ${ }^{a}$ and Andre Sternbeck ${ }^{c}$}

${ }^{a}$ Humboldt Universität zu Berlin, Institut für Physik, 12489 Berlin, Germany

${ }^{b}$ Max-Planck-Institut für Meteorologie, 20146 Hamburg, Germany

${ }^{c}$ CSSM, School of Chemistry \& Physics, University of Adelaide, SA 5005, Australia

E-mail: aivoigtephysik.hu-berlin.de, aiko.voigtezmaw.de,

ilgenfri@physik.hu-berlin.de mmpephysik.hu-berlin.de.

andre.sternbeck@adelaide.edu.au

\begin{abstract}
We study the infrared behaviour of lattice $S U$ (3) Yang-Mills theory in Coulomb gauge in terms of the ghost propagator, the Coulomb potential and the transversal and the time-time component of the equal-time gluon propagator. In particular, we focus on the Gribov problem and its impact on the observables. We observe that the simulated annealing method is advantageous for fixing the Coulomb gauge in large volumes. We study finite-size and discretization effects. While finitesize effects can be controlled by the cone cut, and the ghost propagator and the Coulomb potential become scaling functions with the cylinder cut, the equal-time gluon propagator does not show scaling in the considered range of the inverse coupling constant. The ghost propagator is infrared enhanced. The Coulomb potential is now extended to considerably lower momenta and shows a more complicated infrared regime. The Coulomb string tension satisfies Zwanziger's inequality, but its estimate can be considered only preliminary because of the systematic Gribov effect that is particularly strong for the Coulomb potential.
\end{abstract}

The XXV International Symposium on Lattice Field Theory

July 30-4 August 2007

Regensburg, Germany

\footnotetext{
${ }^{*}$ Speaker.
} 


\section{Motivation and Introduction}

According to the Gribov-Zwanziger scenario, confinement is related to the behaviour of certain gauge-variant two-point functions at small momenta. This is not only true for the Landau gauge. For the Coulomb gauge, similar predictions [1] exist: the transversal component $D_{t r}$ of the equaltime gluon propagator ${ }^{1}$

$$
D_{i j}^{a b}(\vec{q})=\left\langle\tilde{A}_{i}^{a}(\vec{k}) \tilde{A}_{j}^{b}(-\vec{k})\right\rangle=\delta^{a b}\left(\delta_{i j}-\frac{q_{i} q_{j}}{\vec{q}^{2}}\right) D_{t r}\left(q^{2}\right)
$$

should vanish in the limit $q \rightarrow 0$, whereas the ghost propagator $G$, defined by

$$
G^{a b}(\vec{q})=\frac{1}{L^{3}} \sum_{\vec{x}, \vec{y}}\left\langle e^{i \vec{k} \cdot(\vec{x}-\vec{y})}\left[M^{-1}\right]_{\vec{x} \vec{y}}^{a b}\right\rangle=\delta^{a b} G\left(q^{2}\right)
$$

is infrared enhanced ${ }^{2}$. The physical momentum is given by $q_{i}=(2 / a) \cdot \sin \left(\pi k_{i} / L\right)$ with Fourier momenta $k_{i}=-L+1, \ldots, L$. $M$ denotes the Faddeev-Popov operator whose lattice version is given by

$$
M_{\vec{x} \vec{y}}^{a b}=\sum_{i=1}^{3} \mathfrak{R e} \operatorname{Tr}\left[\left\{T^{a}, T^{b}\right\}\left(U_{\vec{x}, i}+U_{\vec{x}-\vec{i}, i}\right) \delta_{\vec{x} \vec{y}}-2 T^{b} T^{a} U_{\vec{x}, i} \delta_{\vec{x}+\vec{i}, \vec{y}}-2 T^{a} T^{b} U_{\vec{x}-\vec{i}, i} \delta_{\vec{x}-\vec{i}, \vec{y}}\right]
$$

Moreover, Zwanziger showed that the instantaneous Coulomb potential $V_{\text {coul }}(r=|\vec{x}-\vec{y}|)$, which appears in the Coulomb gauge Hamiltonian through the elimination of longitudinal gluons and which is related to the Faddeev-Popov operator by

$$
\delta^{a b} V_{\text {coul }}\left(q^{2}\right)=\frac{1}{L^{3}} \sum_{\vec{x}, \vec{y}}\left\langle e^{i \vec{k} \cdot(\vec{x}-\vec{y})}\left[M^{-1}(-\triangle) M^{-1}\right]_{\vec{x} \vec{y}}^{a b}\right\rangle,
$$

has to linearly rise with distance in real space. This is a necessary condition to satisfy confinement usually defined through an area law for Euclidean Wilson loops. The latter translates to a linearly rising bound state potential $V_{\text {wilson }}(r)$. More precisely, Zwanziger's inequality [2] says that $V_{\text {wilson }}(r) \leq-\frac{4}{3} V_{\text {coul }}(r)$ for large distances $r$. Zwanziger and Cucchieri pointed out that the timetime component of the equal-time gluon propagator $D_{44}^{a b}(\vec{q})=\delta^{a b} D_{44}\left(q^{2}\right)$ is also related to the instantaneous Coulomb potential, i.e. $V_{\text {coul }}(r) \simeq\left\langle A_{4}^{a}(\vec{x}) A_{4}^{a}(\vec{y})\right\rangle$ might serve as an useful estimate of the Coulomb potential [3]. We have tested these statements by means of lattice calculations in $S U$ (3) Yang-Mills theory simulated with Wilson's plaquette action. During this conference we learned about a similar investigation carried out by Nakagawa et al. [4].

\section{Details of the simulation}

A summary of the lattices and parameters under investigation is given in Tab. 1. We used two different methods to fix the gauge. The overrelaxation algorithm with $\omega=1.70$ on small and $\omega=1.60$ on large lattices is compared with the simulated annealing method (with $T_{\text {init }}=0.45$ and

\footnotetext{
${ }^{1}$ Here and in the following, the norm of the spatial vector $\vec{q}=\left(q_{1}, q_{2}, q_{3}\right)$ is abbreviated by $q=|\vec{q}|$.

${ }^{2}$ We use the shorthand notation $\vec{k} \cdot \vec{x}=\sum_{i=1}^{3} 2 \pi k_{i} x_{i} / L_{i}$.
} 
$T_{\text {final }}=0.01$ as initial and final temperatures, 1500 compound sweeps at linearly decreasing temperature, each consisting of one heatbath and three microcanonical sweeps, followed by obligatory finalizing overrelaxation). We investigated the Gribov effect by means of the first copy (fc) - best copy (bc) strategy that was used in Landau gauge studies [5]. Since this approach is very demanding in terms of computing time, we have restricted the study of the Gribov problem to smaller lattices with $L^{4} \leq 24^{4}$. On larger lattices only one gauge copy per configuration was generated by means of the simulated annealing algorithm. The bare ghost and equal-time gluon propagator are multiplicatively renormalized at a scale $\mu^{2}=9 \mathrm{GeV}^{2}$. In the case of the Coulomb potential, we use an ultraviolet fit to Eq. 4.1 to fix the physical scale in y-direction.

\begin{tabular}{|cccccccr|}
\hline$L^{4}$ & $\beta$ & $a^{-1}[\mathrm{GeV}]$ & $a[\mathrm{fm}]$ & $V\left[\mathrm{fm}^{4}\right]$ & $\#$ conf & $N_{c p}^{S A}$ & $N_{c p}^{O R}$ \\
\hline \hline $12^{4}$ & 5.8 & 1.446 & 0.1364 & $1.64^{4}$ & 100 & 10 & 1 \\
$16^{4}$ & 5.8 & 1.446 & 0.1364 & $2.18^{4}$ & 40 & 15 & 1 \\
$24^{4}$ & 5.8 & 1.446 & 0.1364 & $3.27^{4}$ & 30 & 20 & 1 \\
$32^{4}$ & 5.8 & 1.446 & 0.1364 & $4.36^{4}$ & 30 & 1 & - \\
$48^{4}$ & 5.8 & 1.446 & 0.1364 & $6.55^{4}$ & 20 & 1 & - \\
\hline \hline $12^{4}$ & 6.0 & 2.118 & 0.0932 & $1.12^{4}$ & 100 & 20 & 20 \\
$16^{4}$ & 6.0 & 2.118 & 0.0932 & $1.49^{4}$ & 60 & 30 & 30 \\
$24^{4}$ & 6.0 & 2.118 & 0.0932 & $2.24^{4}$ & 40 & 40 & 40 \\
$32^{4}$ & 6.0 & 2.118 & 0.0932 & $2.98^{4}$ & 30 & 1 & - \\
$48^{4}$ & 6.0 & 2.118 & 0.0932 & $4.48^{4}$ & 20 & 1 & - \\
\hline \hline $12^{4}$ & 6.2 & 2.914 & 0.0677 & $0.81^{4}$ & 100 & 10 & 1 \\
$16^{4}$ & 6.2 & 2.914 & 0.0677 & $1.08^{4}$ & 40 & 15 & 1 \\
$24^{4}$ & 6.2 & 2.914 & 0.0677 & $1.62^{4}$ & 30 & 20 & 1 \\
$32^{4}$ & 6.2 & 2.914 & 0.0677 & $2.17^{4}$ & 20 & 1 & - \\
\hline
\end{tabular}

Table 1: Ensembles and corresponding parameters as used in our investigation. $N_{c p}^{S A}$ and $N_{c p}^{O R}$ indicate the number of gauge copies per configuration generated with simulated annealing and overrelaxation, respectively.

\section{Gauge fixing}

Gauge fixing is performed by adjusting the gauge transformations $g(\vec{x}, t) \in S U(3)$ separately on each time-slice $t$ such that

$$
F_{U}[g]=\frac{1}{3 L^{3}} \sum_{\vec{x}} \sum_{i=1}^{3} \mathfrak{R e} \operatorname{Tr}\left(\mathbb{1}-{ }^{g} U_{(\vec{x}, t), i}\right) \rightarrow \text { minimum }
$$

The measurement of the $D_{44}$ propagator requires also to fix the remnant global gauge freedom with the temporal links involved, i. e. $U_{(\vec{x}, t), 4} \rightarrow \tilde{g}_{t} U_{(\vec{x}, t), 4} \tilde{g}_{t+1}$. A possible Gribov effect on $D_{44}$ related to an ambiguity in fixing the remnant gauge freedom was not studied. Note also that minimizing $F_{U}[g]$ on the largest lattices was faced with severe critical slowing-down. About $5 \%$ of the timeslices did not converge within 40000 (finalizing) overrelaxation steps. For these time-slices, the entire gauge fixing procedure was repeated until gauge fixing could be successfully accomplished. 

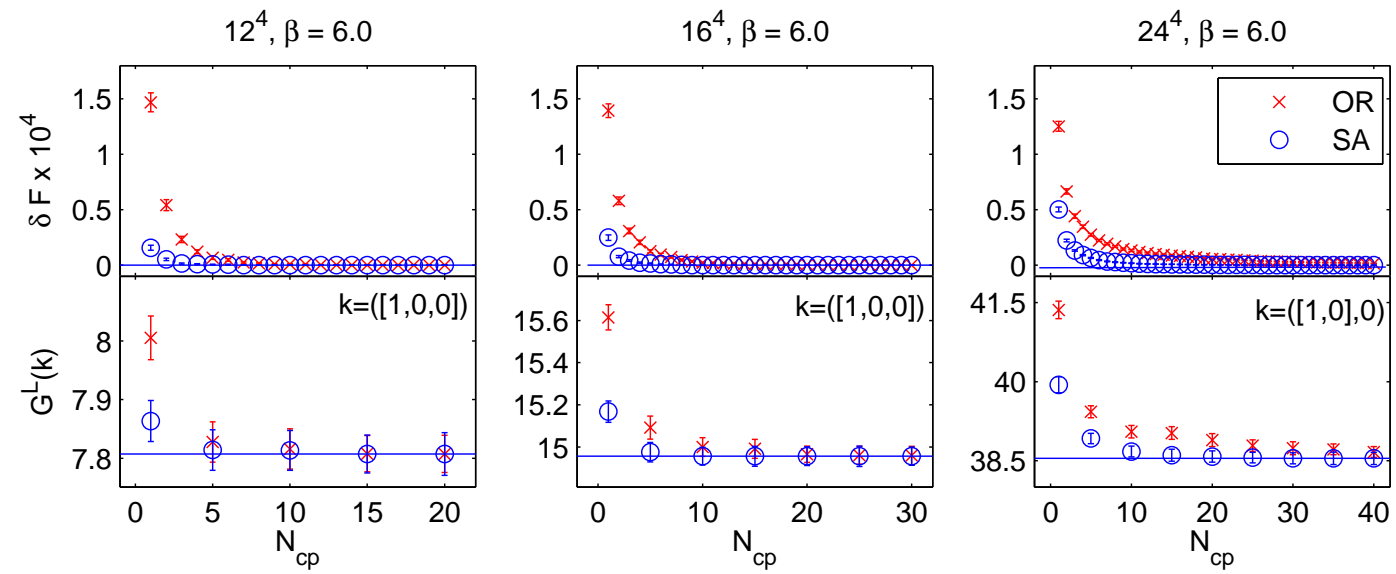

Figure 1: The Gribov ambiguity for different lattice sizes with OR and SA gauge fixing. Upper panels: The convergence of the relative difference $\delta F=1-F^{c b c} / F^{b c}$ between the currently best functional value $F^{c b c}$ after $N_{c p}$ copies and the overall best value $F^{b c}$. Lower panels: the bare ghost propagator at the smallest lattice momentum measured for the currently best gauge copy after $N_{c p}$ copies.

\section{Results}

\subsection{Gribov effect and its impact on the observables}

Following the fc-bc strategy, we have first checked the number of gauge copies necessary to find a functional value $F$ close to the global maximum. This was done separately for both gauge fixing methods. For details of this strategy, we refer to analogous investigations in the Landau gauge [5]. The result is shown in Fig. 1 and points out that simulated annealing clearly needs less gauge copies to assure the convergence of the functional and hence of the observables. Therefore, simulated annealing should be the method of choice, in particular when going to larger lattices. Fig. 2 illustrates the impact of the Gribov effect by comparing the observables measured on the first overrelaxation gauge copy and on the best out of $N_{c p}^{S A}$ simulated annealing gauge copies. As already seen in Landau gauge, the infrared ghost propagator is systematically overestimated by about 5\%. The Coulomb potential shows a similar behaviour, but now the first overrelaxation copy overestimates the Coulomb potential by up to $100 \%$. The equal-time gluon propagator is less affected by the Gribov effect. Its transversal component shows a very weak (1\%) dependence on the Gribov copy in the infrared region. In contrast, the time-time component seems to be underestimated systematically for large momenta.

\subsection{Finite size and discretization effects}

We worked with a large number of lattice sizes and values of the inverse coupling $\beta$ in order to explore finite-size and discretization effects. We found that finite-size effects are visible but can be efficiently removed by the cone cut (see [5] and references therein). Fig. 3 illustrates this for the transversal equal-time gluon propagator. Similarly, the cylinder cut minimizes discretization effects in the cases of the ghost propagator (see Fig. 4) and the Coulomb potential. More problematic is that we find the transversal and the time-time gluon propagator showing strong, systematic discretization effects. This has also been found by Nakagawa et al. [4]. 

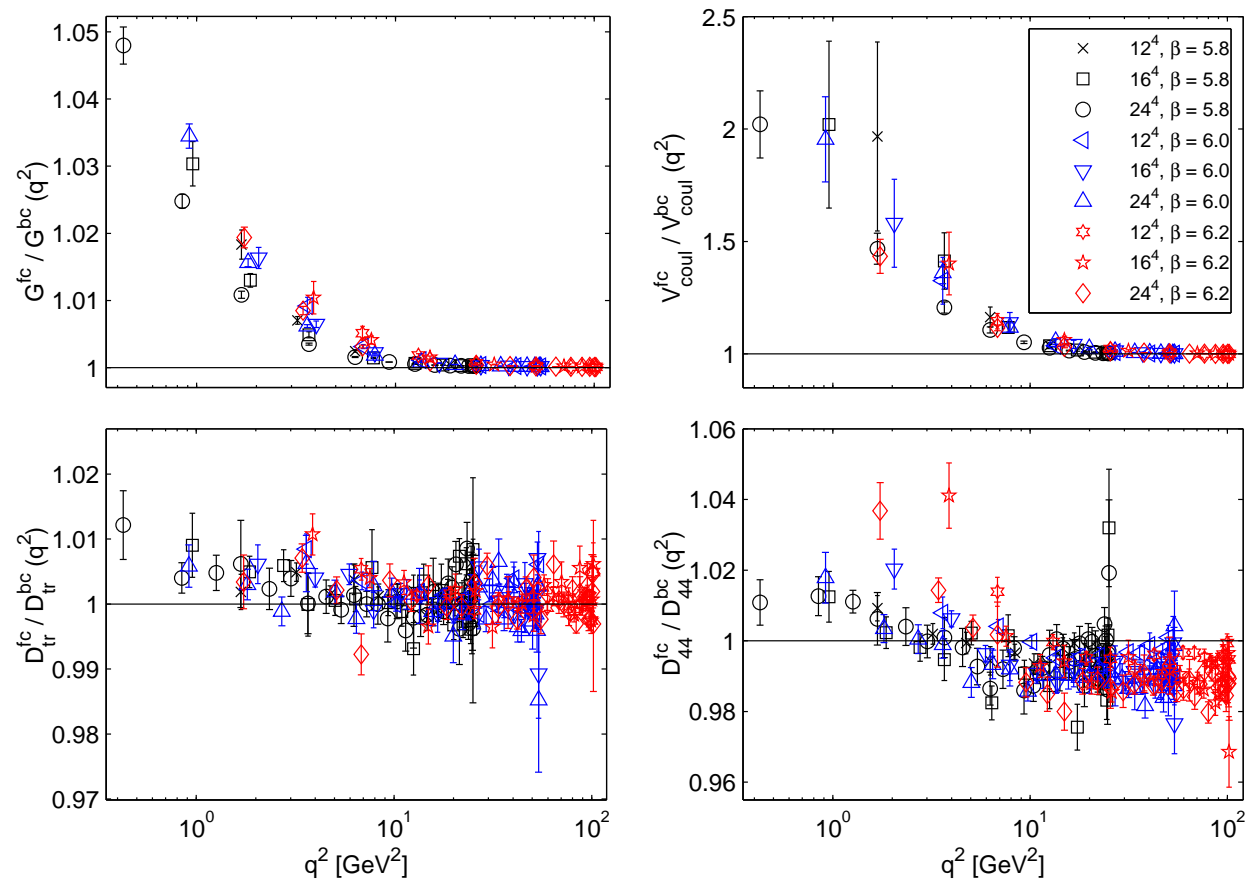

Figure 2: Gribov effect on the propagators and the Coulomb potential: ratios of measured quantites as measured on the first OR copy and on the best of $N_{c p}^{S A}$ SA copies.
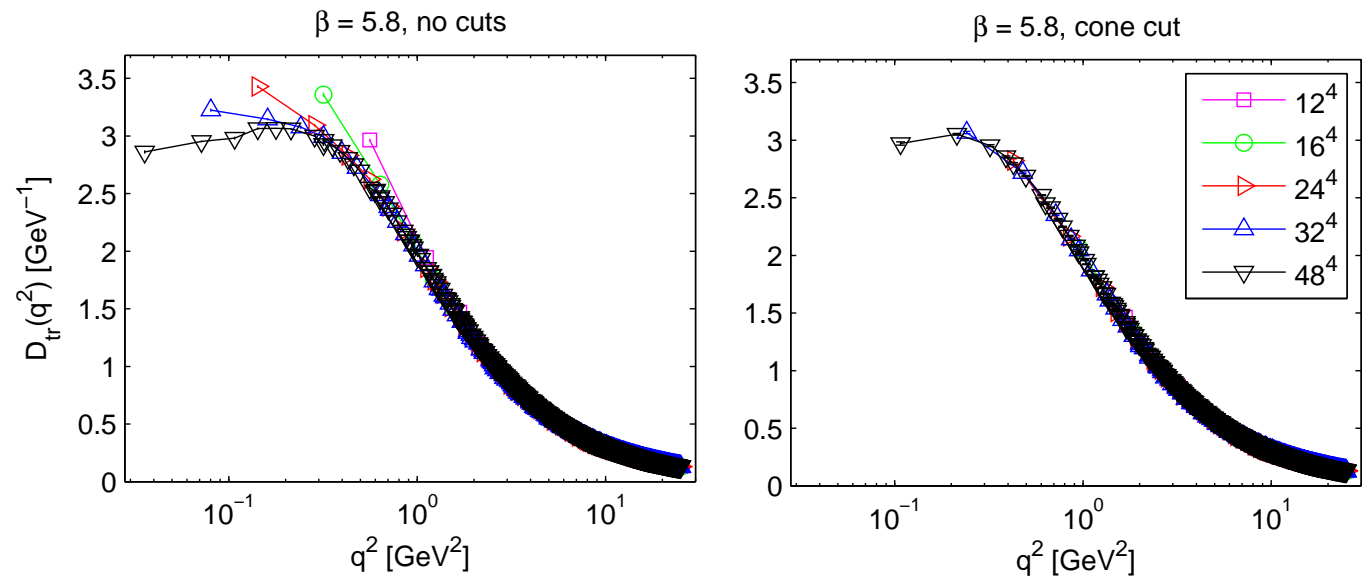

Figure 3: The finite-size effects (left) for the transversal gluon propagator, cured (right) by the cone cut.

\subsection{Infrared behaviour of the lattice observables}

All statements in this section rely on measurements on large lattices using a single ("first") simulated annealing gauge copy and an appropriate combination of the cylinder and the cone cut. Since the equal-time gluon propagator does not show scaling for $\beta=5.8, \ldots, 6.2$ we cannot give reliable statements concerning its behaviour in the infrared regime. However, the turnover of the transversal component for the smallest $\beta=5.8$ and $q^{2} \leq 0.2 \mathrm{GeV}^{2}$ is in agreement with a new infrared regime of the Coulomb potential observed for momenta below this value. The ghost propagator is infrared enhanced. An infrared fit of the ghost propagator to $q^{2} \cdot G\left(q^{2}\right) \simeq q^{-2 \alpha}$ for momenta 

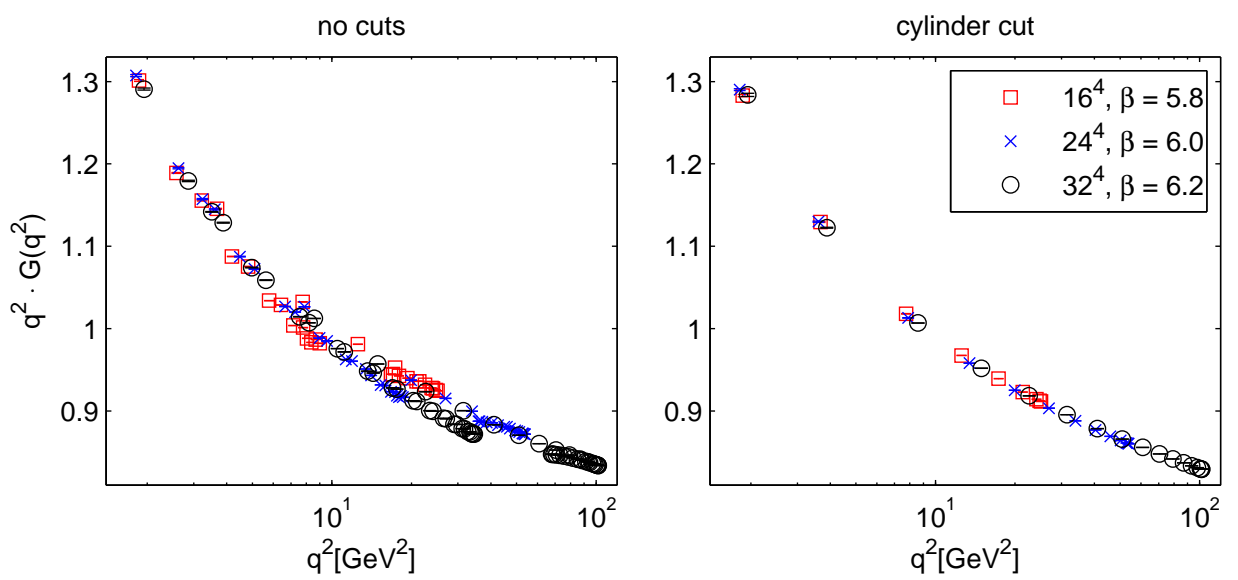

Figure 4: Discretization effects (left) of the ghost dressing function, cured (right) by the cylinder cut.
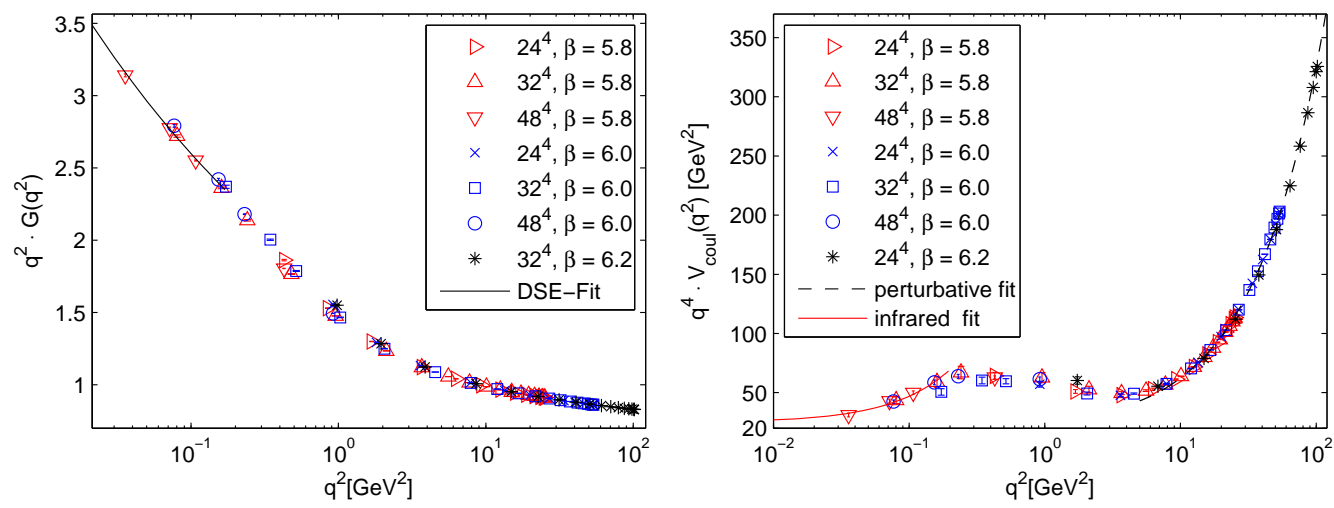

Figure 5: Left: the ghost propagator diverging stronger than $1 / q^{2}$ in the infrared, with a fit giving a too small infrared exponent. Right: the Fourier-transformed Coulomb potential times $q^{4}$, revealing the onset of a new infrared regime for $q^{2}<0.2 \mathrm{GeV}^{2}$, with a fit giving $\sigma_{\text {coul }}$.

$q^{2} \leq 0.17 \mathrm{GeV}^{2}$ gives $\alpha=0.19(2)$. This is by far too small compared to the predictions of DSE studies [6] and, together with the high value of $\chi^{2} /$ d.o.f. $\simeq 12$, indicates that the momentum region accessible in our study is not yet in the scope of the envisaged infrared power laws. We should also admit that our fit is clearly limited by the small number of points available in this momentum region.

The Coulomb potential is infrared divergent. Plotting $q^{4} \cdot V_{\text {coul }}\left(q^{2}\right)$ reveals that a new infrared regime is opened for momenta smaller than $q^{2} \sim 0.2 \mathrm{GeV}^{2}$. After an intermediate plateau is reached, $q^{4} \cdot V_{\text {coul }}\left(q^{2}\right)$ decreases further with decreasing momenta. We used this momentum region to estimate the Coulomb string tension to $\sigma_{\text {coul }}=(5.0 \pm 1.3) \sigma_{\text {wilson }}$, even higher than Zwanziger's inequality requires. To do so, first an ultraviolet fit

$$
q^{4} \cdot V_{\text {coul }}\left(q^{2}\right)=\frac{192 \pi^{2}}{121} \frac{q^{2}}{\ln \left(q^{2} / \Lambda_{\text {coul }}^{2}\right)} \quad \text { for } q^{2} \geq 20 \mathrm{GeV}^{2}, \Lambda_{\text {coul }}=0.9(3) \mathrm{GeV}
$$

and an infrared fit representing a sum of a constant force and a $1 / q^{2}$ Coulomb law,

$$
q^{4} \cdot V_{\text {coul }}\left(q^{2}\right)=8 \pi \sigma_{\text {coul }}+4 \pi C q^{2} \quad \text { for } q^{2} \leq 0.16 \mathrm{GeV}^{2}, C=17.8 \pm 5.9
$$


had to be performed. Note that our value of the Coulomb string tension does not take into account the strong Gribov effect on the Coulomb potential in the infrared. It is strictly based on data for a single simulated annealing copy. Hence, the Coulomb string tension might be overestimated by a factor of two. This would be in better agreement with values obtained by measurements of partial Polyakov line correlators [7]. We also stress that our result is in contrast to $S U(2)$ studies that had suggested $\sigma_{\text {coul }} \simeq \sigma_{\text {wilson }}[8]$. In view of the different results for the Coulomb potential and the time-time component of the gluon propagator, we conclude that the latter should not be used to estimate the Coulomb potential. The issue of non-scaling of the equal-time gluon propagator is currently investigated and might be solved by a more advanced renormalization procedure.

\section{Acknowledgements}

Part of this work is supported by DFG under contract FOR 465 (Forschergruppe Gitter - Hadronen Phänomenologie), and by the Australian Research Council. A major part of the simulations have been done on the IBM pSeries 690 at HLRN. We thank Hinnerk Stüben for contributing parts of the code.

\section{References}

[1] V. N. Gribov, Quantization of non-Abelian gauge theories, Nucl. Phys. B139 (1978) 1

D. Zwanziger, Renormalization in the Coulomb gauge and order parameter for confinement in $Q C D$, Nucl. Phys. B518 (1998) 237

[2] D. Zwanziger, No confinement without Coulomb confinement, Phys. Rev. Lett. 90 (2003) 102001 [hep-lat/0209105]

[3] A. Cucchieri and D. Zwanziger, Renormalization group calculation of color Coulomb potential, Phys. Rev. D65 (2002) 014002 [hep-th/ 0008248 ]

A. Cucchieri and D. Zwanziger, Numerical study of gluon propagator and confinement scenario in minimal Coulomb gauge, Phys. Rev. D65 (2002) 014001 [hep-lat / 0008026 ]

[4] Y. Nakagawa, A. Nakamura, T. Saito, and H. Toki, PoS LAT2007 to be published

[5] A. Sternbeck, E. -M. Ilgenfritz, M. Müller-Preussker, and A. Schiller, Towards the infrared limit in SU(3) Landau gauge lattice gluodynamics, Phys. Rev. D72 (2005) 014507 [hep-lat / 0506007 ]

[6] D. Epple, H. Reinhardt, and W. Schleifenbaum, Confining solution of the Dyson-Schwinger equations in Coulomb gauge, Phys. Rev. D75 (2007) 045011 [hep-th / 0 6122 41]

W. Schleifenbaum, M. Leder, and H. Reinhardt, H., Infrared analysis of propagators and vertices of Yang-Mills theory in Landau and Coulomb gauge, Phys. Rev., D73 (2006) 125019

[hep-th/0605115]

[7] A. Nakamura and T. Saito, Color confinement in Coulomb gauge QCD, Prog. Theor. Phys. 115 (2006) 189 [hep-lat / 0512042]

Y. Nakagawa, A. Nakamura, T. Saito, H. Toki, and D. Zwanziger, Properties of color-Coulomb string tension, Phys. Rev. D73 (2006) 094504 [hep-lat/ 0603010 ]

[8] A. Cucchieri and D. Zwanziger, Gluon propagator and confinement scenario in Coulomb gauge, Nucl. Phys. Proc. Suppl. 119 (2003) 727 [hep-lat/0209068]

K. Langfeld L. Moyaerts, Propagators in Coulomb gauge from SU(2) lattice gauge theory, Phys. Rev. D70 (2004) 074507 [hep-lat / 0406024 ] 